\title{
Can the use of an age-adjusted D-dimer cut-off value help in our diagnosis of suspected pulmonary embolism? 裖
}

\author{
Authors: Jonathan Dutton, ${ }^{A}$ Martin Dachsel ${ }^{B}$ and Rachel Crane ${ }^{C}$
}

Safe exclusion of pulmonary embolism (PE) is a common problem in acute medicine. Common care pathways usually involve the use of a pre-test probability score with a D-dimer test to aid clinical decision-making. Unfortunately, the specificity of many D-dimer assays decreases with age. This study investigates the role of an age-adjusted D-dimer of $5 \mathrm{x}$ patient's age when the conventional cut-off of the assay is $230 \mathrm{ng} / \mathrm{mL}$. Data was collected retrospectively over a 12-month period from patients who went on to have either computed tomography pulmonary angiography (CTPA) or pulmonary ventilation/perfusion (V/Q) imaging. D-dimers in patients with low or moderate Wells score were analysed for both conventional and age-adjusted cut-offs. The use of an adjusted D-dimer showed a sensitivity of $0.97(95 \%$ CI 0.9-1.0) while the specificity increased from 0.07 (95\% CI 0.04-0.11) for the conventional cut-off to 0.32 (95\% CI 0.27-0.38) for the age-adjusted cut-off. Using a $5 \mathrm{x}$ patient's age-adjusted D-dimer cut-off is both safe and showed an increased specificity comparable to those published previously on other D-dimer assays.

KEYWORDS: Pulmonary embolism, D-dimer, acute medicine

\section{Introduction}

Chest pain is a common presenting complaint to emergency and acute medicine departments and constitutes a large proportion of patients being admitted to the medical take; ${ }^{1} 10-15 \%$ of these patients are evaluated for pulmonary embolism (PE). ${ }^{2}$ The annual incidence rises with age from less than five cases per 100,000 in children to around 500 cases per 100,000 in older adults over 80 years of age. ${ }^{3}$

Pathways to investigate PE include the combined use of a pre-test probability (PTP) score and high sensitive D-dimer test (Supplementary file 1). D-dimer is a fibrin degradation product, which has a high negative predictive value for PE. ${ }^{4}$ Unfortunately,

Authors: Afoundation doctor, Surrey and Sussex Healthcare Trust, East Surrey Hospital, Redhill, UK; ${ }^{\text {B }}$ consultant of acute medicine, Surrey and Sussex Healthcare Trust, East Surrey Hospital, Redhill,

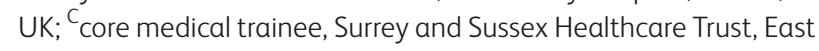
Surrey Hospital, Redhill, UK
D-dimer values increase with advancing age, leading to a decreased specificity and therefore a reduced ability to exclude PE in older adults. ${ }^{5}$

The rising incidence coupled with a decreased ability to detect true negative patients through D-dimer measurements result in an increasing number of patients with further diagnostic tests performed.

A number of studies have been published with a conventional cut-off value of $500 \mathrm{ng} / \mathrm{mL}$ and an age-adjusted D-dimer cut-off value of age (in years) $\times 10.6,7$

In this study we are investigating the potential effect of excluding PE with a common, but different conventional D-dimer cut-off $(230 \mathrm{ng} / \mathrm{mL}$ ) and an age-adjusted D-dimer of age (in years) $\times 5$.

\section{Method}

Data was collected retrospectively as a quality improvement project in a district general hospital for a selected consecutive cohort of patients over the 12-month period between April 2016 and March 2017, for whom there was a clinical suspicion of PE and who underwent imaging to confirm or exclude the diagnosis. The local PACS (Picture achieving and communication system) department provided a list of all patients over the age of 50 years with a computed tomography pulmonary angiogram (CTPA) or ventilation-perfusion (V/Q) scan requested in the first 24 hours of their admission via either the emergency department or acute medical unit ( $n=593)$.

Patients were excluded from further analysis if they presented with a high PTP ( $n=225$ ), with uncompleted scans $(n=1)$ and with no $D$-dimer assay performed $(n=38)$. Twenty-nine patients had no PTP recorded; however, they were included into the study since they had similar incidence of PE as the low/intermediate risk group (Table 1). In total, 329 patients were included in the final analysis.

\section{Table 1. Pre-test probability and pulmonary} embolism (PE) diagnosis

\begin{tabular}{|c|c|c|c|}
\hline \multicolumn{2}{|c|}{ Wells Score } & PE & No PE \\
\hline High & $n=224(37.9 \%)$ & $72(32.1 \%)$ & $152(67.9 \%)$ \\
\hline Intermediate & $\mathrm{n}=262(44.3 \%)$ & $55(21.0 \%)$ & $207(79.0 \%)$ \\
\hline Low & $\mathrm{n}=76(12.9 \%)$ & $15(19.7 \%)$ & $61(80.3 \%)$ \\
\hline Not documented & $\mathrm{n}=29(4.9 \%)$ & $6(20.7 \%)$ & $23(79.3 \%)$ \\
\hline
\end{tabular}




\begin{tabular}{|c|c|c|c|}
\hline & PE & No PE & $\begin{array}{l}\text { Significance } \\
\text { level }\end{array}$ \\
\hline Number & 69 & 260 & \\
\hline Age & $71[64-82]$ & 71 [63-79] & $p=0.40$ \\
\hline Female & $49.3 \%$ & $54.6 \%$ & $p=0.51$ \\
\hline D-dimer & $1584[976-3,773]$ & 505 [320-912] & $p<0.001$ \\
\hline
\end{tabular}

Pre-test probability (PTP) using a 3-level Wells score and D-dimer (HemosILTM D-dimer assay, cut-off $230 \mathrm{ng} / \mathrm{mL}$ ) were recorded.

Radiology results were reported by consultant radiologists and in case of simultaneous V/Q scan and CTPA, the CTPA result was used as it is considered gold standard.

The D-dimer result was interpreted according to our conventional cut-off ( $230 \mathrm{ng} / \mathrm{mL}$ ). In addition, an age-adjusted cut-off was analysed, which was considered negative if $\mathrm{D}$-dimer was lower than a patient's age (in years) $\times 5$. Age (in years) $\times 5$ was chosen as the age-adjusted cut-off due to our local conventional cut-off being around half as the widely reported cut-off value of $500 \mathrm{ng} / \mathrm{mL}$.

The data was collected and analysed using Microsoft Excel and $R$ (version 3.4.3). Normal distribution was tested with ShapiroWilk test and normal distributed values are shown as mean and standard deviation, otherwise as median and interquartile range. Parametric variables were tested for significant differences with Welch's t-test (if normal distributed), otherwise a Mann-Whitney $U$ test was used. Non-parametric variables were tested with Mann-Whitney $U$ test. Frequencies were analysed with Chisquared test. Sensitivities, specificities, negative predictive values (NPV) and positive predictive values (PPV) are shown as value and 95\% confidence interval (CI).

\section{Results}

During the 12-month study period a total of 329 patients over the age of 50 with low or moderate Wells score were imaged for suspected PE (317 CTPA [98.3\%], 12 V/Q scan [1.7\%]). Sixty-nine patients (21\%) were diagnosed with PE. There were no differences in age or sex distribution; however, the PE group had significantly higher D-dimer levels (Table 2).

Of the 69 PEs identified, the majority affected either the lobar branches $(37.68 \%)$ or the main pulmonary arteries $(37.68 \%)$;
$17.39 \%$ were affecting segmental arteries and $7.24 \%$ the subsegmental branches.

Conventional D-dimer assay was true positive (TP) in 69 patients and true negative (TN) in 18 patients. There were no false negative (FN) results in the cohort, but 242 false positive (FP) results. Age-adjusted D-dimer showed two FN results, the number of TNs increased to 84 patients with subsequent reduction of FP results ( $n=176)$. The full distribution of the two cut-offs for different age decades is shown in Table 3.

The sensitivity of the conventional D-dimer cut-off was 1.00 (95\% CI $0.95-1.00)$ in our cohort with a specificity of 0.07 (95\% CI $0.04-0.11)$. The values for the age-adjusted D-dimer cut-off were 0.97 (95\% CI 0.90-1.00) and 0.32 (95\% CI 0.27-0.38) respectively. Negative predictive value changed little (from 1.00 [95\% CI 0.81-1.00] to 0.98 [95\% CI 0.92-1.00]). The full test statistics for conventional and age-adjusted cut-offs per age decade are shown in Table 4.

The aim for this study was to investigate a $5 \mathrm{x}$ age cut-off; however, calculations were also done for different age-adjusted cut-offs from $3 \times$ patient's age to $10 \times$ patient's age. The test statistics for these age-adjusted cut-offs are shown in Supplementary file 2 .

There were two cases where the age-adjusted strategy would have wrongly resulted in the early exclusion of PE: patient 1 ( 82 years old, male, intermediate PTP) was diagnosed with a right-sided lobar PE, that had previously been diagnosed and the patient was already on long-term anticoagulation with warfarin, and patient 2 (73 years old, male, intermediate PTP) was diagnosed with a sub-segmental pulmonary embolus affecting just one laterality.

\section{Discussion}

This retrospective study was performed to assess the use of an age-adjusted D-dimer using the Instrumentation Lab D-dimer assay with a reported conventional cut-off value of $230 \mathrm{ng} /$ $\mathrm{mL}$. Most published studies reporting on age-adjusted D-dimer used an assay with a conventional cut-off of $500 \mathrm{ng} / \mathrm{mL}$. ${ }^{6,8,9}$ Considering that our conventional cut-off is approximately half of the previously reported studies, we investigated an age-adjusted cut-off of $5 x$ patient's age.

The sensitivity of our age-adjusted cut-off was $97 \%$, similar to studies by Douma et al ${ }^{6}$ and Righini et al. ${ }^{9}$ Increasing the cut-off to $6 \mathrm{x}$ patient's age showed a similar sensitivity with a much larger gain in specificity than reported elsewhere. ${ }^{6,8,9}$ Reducing the age-adjusted cut-off to $4 \mathrm{x}$ patient's age increased the sensitivity minimally but halved the specificity with no change of NPV.

Table 3. Distribution of D-dimer results for conventional and age-adjusted cut-offs and patient's age per decade

\begin{tabular}{|c|c|c|c|c|c|c|c|c|c|}
\hline \multirow[b]{2}{*}{ Age group } & \multirow[b]{2}{*}{ Number } & \multicolumn{4}{|c|}{ Conventional D-dimer } & \multicolumn{4}{|c|}{ Age-adjusted D-dimer } \\
\hline & & TP & FP & $\mathrm{FN}$ & $\mathrm{TN}$ & TP & FP & FN & $\mathrm{TN}$ \\
\hline All ages & 329 & 69 & 242 & 0 & 18 & 67 & 176 & 2 & 84 \\
\hline $50-59$ & 59 & 11 & 38 & 0 & 10 & 11 & 32 & 0 & 16 \\
\hline $60-69$ & 90 & 18 & 68 & 0 & 4 & 18 & 52 & 0 & 20 \\
\hline 70-79 & 96 & 18 & 75 & 0 & 3 & 17 & 48 & 1 & 30 \\
\hline $80-89$ & 68 & 17 & 50 & 0 & 1 & 16 & 37 & 1 & 14 \\
\hline $90-99$ & 16 & 5 & 11 & 0 & 0 & 5 & 7 & 0 & 4 \\
\hline
\end{tabular}


Table 4. Sensitivity, specificity, positive predictive value (PPV) and negative predictive value (NPV) of conventional and age adjusted D-dimer (value and $95 \%$ confidence interval)

\begin{tabular}{|c|c|c|c|c|c|c|c|c|c|}
\hline \multirow[b]{2}{*}{ Age group } & \multirow[b]{2}{*}{ Number } & \multicolumn{4}{|c|}{ Conventional D-dimer } & \multicolumn{4}{|c|}{ Age-adjusted D-dimer } \\
\hline & & Sensitivity & Specificity & PPV & NPV & Sensitivity & Specificity & PPV & NPV \\
\hline All ages & 329 & $\begin{array}{l}1.00 \\
(0.95-1.00)\end{array}$ & $\begin{array}{l}0.07 \\
(0.04-0.11)\end{array}$ & $\begin{array}{l}0.22 \\
(0.18-0.27)\end{array}$ & $\begin{array}{l}1.00 \\
(0.81-1.00)\end{array}$ & $\begin{array}{l}0.97 \\
(0.90-1.00)\end{array}$ & $\begin{array}{l}0.32 \\
(0.27-0.38)\end{array}$ & $\begin{array}{l}0.28 \\
(0.22-0.34)\end{array}$ & $\begin{array}{l}0.98 \\
(0.92-1.00)\end{array}$ \\
\hline $50-59$ & 59 & $\begin{array}{l}1.00 \\
(0.72-1.00)\end{array}$ & $\begin{array}{l}0.21 \\
(0.10-0.35)\end{array}$ & $\begin{array}{l}0.22 \\
(0.12-0.37)\end{array}$ & $\begin{array}{l}1.00 \\
(0.69-1.00)\end{array}$ & $\begin{array}{l}1.00 \\
(0.72-1.00)\end{array}$ & $\begin{array}{l}0.33 \\
(0.20-0.48)\end{array}$ & $\begin{array}{l}0.26 \\
(0.14-0.41)\end{array}$ & $\begin{array}{l}1.00 \\
(0.79-1.00)\end{array}$ \\
\hline $60-69$ & 90 & $\begin{array}{l}1.00 \\
(0.81-1.00)\end{array}$ & $\begin{array}{l}0.06 \\
(0.02-0.14)\end{array}$ & $\begin{array}{l}0.21 \\
(0.13-0.31)\end{array}$ & $\begin{array}{l}1.00 \\
(0.40-1.00)\end{array}$ & $\begin{array}{l}1.00 \\
(0.81-1.00)\end{array}$ & $\begin{array}{l}0.28 \\
(0.18-0.40)\end{array}$ & $\begin{array}{l}0.26 \\
(0.16-0.38)\end{array}$ & $\begin{array}{l}1.00 \\
(0.83-1.00)\end{array}$ \\
\hline 70-79 & 96 & $\begin{array}{l}1.00 \\
(0.81-1.00)\end{array}$ & $\begin{array}{l}0.04 \\
(0.01-0.11)\end{array}$ & $\begin{array}{l}0.19 \\
(0.12-0.29)\end{array}$ & $\begin{array}{l}1.00 \\
(0.29-1.00)\end{array}$ & $\begin{array}{l}0.94 \\
(0.73-1.00)\end{array}$ & $\begin{array}{l}0.38 \\
(0.28-0.50)\end{array}$ & $\begin{array}{l}0.26 \\
(0.16-0.39)\end{array}$ & $\begin{array}{l}0.97 \\
(0.83-1.00)\end{array}$ \\
\hline $80-89$ & 68 & $\begin{array}{l}1.00 \\
(0.80-1.00)\end{array}$ & $\begin{array}{l}0.02 \\
(0.00-0.10)\end{array}$ & $\begin{array}{l}0.25 \\
(0.16-0.37)\end{array}$ & $\begin{array}{l}1.00 \\
(0.02-1.00)\end{array}$ & $\begin{array}{l}0.94 \\
(0.71-1.00)\end{array}$ & $\begin{array}{l}0.27 \\
(0.16-0.42)\end{array}$ & $\begin{array}{l}0.30 \\
(0.18-0.44)\end{array}$ & $\begin{array}{l}0.93 \\
(0.68-1.00)\end{array}$ \\
\hline $90-99$ & 16 & $\begin{array}{l}1.00 \\
(0.48-1.00)\end{array}$ & $\begin{array}{l}0.00 \\
(0.00-0.28)\end{array}$ & $\begin{array}{l}0.31 \\
(0.11-0.59)\end{array}$ & $\begin{array}{l}n / a \\
(0.00-1.00)\end{array}$ & $\begin{array}{l}1.00 \\
(0.48-1.00)\end{array}$ & $\begin{array}{l}0.36 \\
(0.11-0.69)\end{array}$ & $\begin{array}{l}0.42 \\
(0.15-0.72)\end{array}$ & $\begin{array}{l}1.00 \\
(0.40-1.00)\end{array}$ \\
\hline
\end{tabular}

Some authors suggest that subsegmental PE are part of the physiological filter function of the lungs and so should not be diagnosed or treated. ${ }^{10}$ By hypothetically excluding 'false negative patients' with subsegmental PE the sensitivity would increase to 0.99 (95\% CI 0.92-1.00).

The largest gain from using an age-adjusted D-dimer is the increase in specificity. Using the conventional cut-off would drop the specificity from approximately $30 \%$ for patients up to 50 years of age, to under $2 \%$ for patients over 80 years of age. Righini et al showed that an age-adjusted D-dimer strategy increased the specificity back to around $30 \% .{ }^{9}$ Our $5 \times$ patient's age-adjusted D-dimer demonstrates specificity to be similar throughout all age groups.

Using an age-adjusted strategy, an additional 67 patients could have had PE excluded without any further imaging. This has the potential to avoid the potential harm associated with the radiation and contrast nephropathies (reported in up to $14 \%$ of patients after CTPA $^{11}$ ) as well as reducing the additional length of stay and further hospital costs associated with these investigations.

Recently, Lapner et al questioned the usefulness of an age-adjusted D-dimer. ${ }^{12}$ They argued that an increase of the conventional cutoff threshold to a 'mean' D-dimer (average D-dimer cut-off of all age groups) or even using an 'inverted' age-adjusted D-dimer will provide similarly high NPVs. This is not surprising, considering the combination of a relatively low prevalence and a highly sensitive test. However, the driving force for using an age-adjusted strategy is the increase in specificity and the decrease of FP results. Testing our cohort against the Lapner et I $^{12}$ study (see Supplementary file 3) showed a reduced specificity in the 'mean' D-dimer cut-off ( 0.21 [95\% CI 0.16-0.27]), while the sensitivity in the 'inverted' group dropped to 0.94 (95\% CI 0.86-0.98).

Another contemporary study by Farm et al investigated ageadjusted D-dimer for four different D-dimer assays and found the use of age-adjusted D-dimer approach improved specificity with maintained sensitivity in all four assays with a substantial decrease in false positives, similar to our study. ${ }^{13}$

\section{Limitations of this study}

Data was collected retrospectively over a 1-year period for those patients investigated with CTPAs and V/Q scans, and as such has the limitations of any retrospective, self-selecting study. There has been no prospective study with this D-dimer assay to our knowledge; however, our sensitivity/specificity and PPV/NPV are in a similar range to studies using the more traditional $500 \mathrm{ng} / \mathrm{mL}$ cut-off. $6,8,9$

The study population was identified via our radiology department. Patients with low or moderate clinical risk scores and a negative D-dimer were not included. Therefore, our PTP is higher than in an unselected population. This shows in the rate of PEs diagnosed, which is higher than in the studies by Righini et al ${ }^{9}$ or Penaloza et al. ${ }^{14}$ In view of this, it is reassuring that the NPV is over $98 \%$. The NPV will be higher in an unselected population with lower PTP. However, the specificity might be lower respectively.

As a next step we would like to perform a prospective multicentre study with this D-dimer assay and age-adjusted D-dimer cut-off.

\section{Conclusion}

Using $5 \times$ patient's age as an age-adjusted D-dimer cut-off seems safe, even in this higher risk population. It can reduce the number of patients investigated for PE by $20 \%$ and has the potential to reduce costs and harm, with the advantage of an equal specificity to the conventional cut-off for younger patients.

\section{Author contributions}

$\mathrm{MD}, \mathrm{RC}$ and JD contributed to the collection of data, data analysis and drafts of the paper at all stages.

\section{Supplementary material}

Supplementary material may be found in the online version of this article at www.clinmed.rcpjournal.org:

S1 - Suggested pathway to investigate for suspected pulmonary embolism.

S2 - Sensitivity, specificity, positive predictive value (PPV) and negative predictive value (NPV) of $3 x$ age to $10 x$ age cut-offs.

S3 - Mean D-dimer and inverted age adjusted D-dimer - sensitivity, specificity, positive predictive value (PPV) and negative predictive value (NPV) of our cohort using the Lapner et al ${ }^{13}$ criteria. 


\section{References}

1 Fothergill NJ, Hunt MT, Touquet R. Audit of patients with chest pain presenting to an accident and emergency department over a 6-month period. Arch Emerg Med 1993;10:155-60.

2 Cayley WE. Diagnosing the cause of chest pain. Am Fam Physician 2005;72:2012-21.

3 White RH. The epidemiology of venous thromboembolism. Circulation 2003;107(23 Suppl 1):I4-8.

4 Di Nisio M, Squizzato A, Rutjes AW et al. Diagnostic accuracy of D-dimer test for exclusion of venous thromboembolism: a systematic review. J Thromb Haemost 2007;5:296-304.

5 Harper PL, Theakston E, Ahmed J, Ockelford P. D-dimer concentration increases with age reducing the clinical value of the D-dimer assay in the elderly. Intern Med 2007;37:607-13.

6 Douma RA, Le Gal G, Söhne M et al. Potential of an age adjusted D-dimer cut-off value to improve the exclusion of pulmonary embolism in older patients: a retrospective analysis of three large cohorts. BMJ 2010;340:c1475.

7 Haas FJ, Schutgens RE, Biesma DH. An age-adapted approach for the use of D-dimers in the exclusion of deep venous thrombosis. Am J Hematol 2009;84:488-91.

8 Douma RA, Tan M, Schutgens REG et al. Using an age-dependent D-dimer cut-off value increases the number of older patients in whom deep vein thrombosis can be safely excluded. Haematologica 2012;97:1507-13.

9 Righini M, Van Es J, Den Exter PL et al. Age-adjusted d-dimer cutoff levels to rule out pulmonary embolism: the ADJUST-PE study. JAMA 2014:311:1117-24.
10 Stein PD, Goodman LR, Hull RD et al. Diagnosis and management of isolated subsegmental pulmonary embolism: review and assessment of the options. Clin Appl Thromb Hemost 2012:18:20-6.

11 Mitchell A, Jones A, Tumlin JA, Kline JA. Prospective study of the incidence of contrast-induced nephropathy among patients evaluated for pulmonary embolism by contrast-enhanced computed tomography. Acad Emerg Med 2012;19:618-25.

12 Lapner S, Julian JA, Linkins LA et al. Questioning the use of an ageadjusted D-dimer threshold to exclude venous thromboembolism: analysis of individual patient data from two diagnostic studies. J Thromb Haemost 2016;14:1953-9.

13 Farm M, Siddiqui A, Onelöv L et al. Age-adjusted D-dimer cut-off leads to more efficient diagnosis of venous thromboembolism in the emergency department: a comparison of four assays. J Thromb Haemost 2018;16:866-75.

14 Penaloza A, Roy PM, Kline ] et al. Performance of age-adjusted D-dimer cut-off to rule out pulmonary emblism. J Thromb Haemost 2012;10:1291-6.

Address for correspondence: Dr Jonathan Dutton, Department of Acute Medicine, East Surrey Hospital, Surrey and Sussex Healthcare, Canada Avenue, Redhill RH1 5RH, UK. Email: jonathan.dutton@doctors.org.uk

\section{Royal College of Physicians}

\section{Physicians and war}

War has been a frequent feature of British life over the 500 years of the Royal College of Physicians' existence, and has an important place in the histories of both the RCP and medicine.

Order by phone or online

Tel +44 (0)20 30751358

or visit www.rcplondon.ac.uk/shop

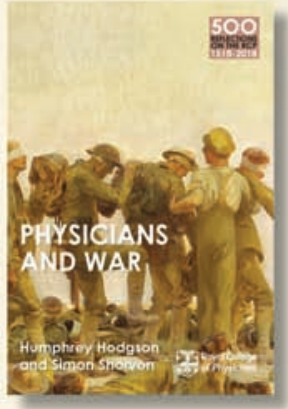

Price: $€ 12$

(Postage and packaging not included)

$10 \%$ discount for fellows and members,

$15 \%$ discount for foundation doctors and students 